\title{
Caminhos Virtuais e Autismo: acesso aos serviços de saúde na perspectiva da Análise de Redes Sociais
}

\author{
Virtual Paths and Autism: access to health services \\ from the perspective of Social Network Analysis
}

Lívia Peluso Rossi ${ }^{1}$

Giovanni Marcos Lovisi ${ }^{1}$

Lucia Abelha ${ }^{1}$

Marcia Gomide ${ }^{1}$
${ }^{1}$ Instituto de Estudos em Saúde Coletiva, Universidade Federal do Rio de Janeiro. Av. Brigadeiro Trimpowsky s/n - Pça da Prefeitura Universitária, Ilha do Fundão. 22713-370 Rio de Janeiro RJ Brasil. livia_peluso@hotmail.com

\begin{abstract}
The prevalence of Autism Spectrum Disorder in the world population and in Brazil is increasing. The Internet has become an important source of information regarding access to health services, including mental health. It remains to be seen if the virtual paths in search of information are related to the outcomes of the line of care for mental disorders advocated by the SUS, such as Autism. Therefore, this article sets out to analyze the virtual network of access to information about care for Autism in the municipality of Rio de Janeiro in 2017 through the perspective of Social Network Analysis. In this regard, virtual data were collected, such as: virtual information sources; care services cited by the virtual sources and type of service (Public, Private or NGOs). Through the use of Gephi software, a sociogram was generated and analyzed. The results point to a predominance of NGO services in the network, greater centrality of degree and power of intermediation in NGO services, in addition to the isolation of public health services. The result implies that the information system about access to public health services for the treatment of ASD is expanded to the general population by contributing to improved access to these services.
\end{abstract}

Key words Autistic disorder, Social networking, Accessibility of health services, Internet
Resumo Constata-se o aumento da prevalência do Transtorno do Espectro Autista na população mundial e no Brasil. A internet tem se constituído importante fonte de informação ao acesso aos serviços de saúde, incluindo a saúde mental. Cabe sabermos se os caminhos virtuais em busca de informação se relacionam aos desfechos da linha de cuidado para transtornos mentais preconizada pelo SUS, como para o Autismo. Portanto, o presente artigo tem como objetivo analisar a rede virtual de acesso a informações sobre atendimento para Autismo no município do Rio de Janeiro em 2017, através da perspectiva da Análise de Rede Sociais. Para isso, foram coletados dados virtuais como: fontes virtuais de informação, serviços de atendimento citados pelas fontes virtuais e tipo de serviço (Público, privado ou ONGs). Através do uso do software Gephi, foi gerado e analisado um sociograma. Os resultados apontam para uma predominância de serviços de ONGs na rede, maior centralidade de grau e poder de intermediação desses serviços, além do isolamento dos serviços públicos de saúde. O resultado pressupõe que o sistema de informações acerca do acesso aos serviços de saúde pública para o tratamento do TEA seja expandido para a população em geral, contribuindo com a melhoria do acesso a esses serviços.

Palavras-chave Transtorno autístico, Rede social, Acesso aos serviços de saúde, Internet 


\section{Introdução}

Nos últimos anos vem-se constatando o aumento da prevalência de Transtorno do Espectro Autista (TEA) na população mundial ${ }^{1}$. Em 2012, foi estimada uma prevalência de TEA de 14,6\% em 1.000 , ou seja, o equivalente a uma criança em cada 68, com 8 anos de idade, nos Estados Uni$\mathrm{dos}^{1}$. Mais recentemente foi estimada uma prevalência de 2,47 \% entre crianças e adolescentes dos EUA, em 2014-2016².

No Brasil, existem poucos estudos que abordam a Epidemiologia para TEA ${ }^{3}$. Dentre eles, um que estimou uma frequência de TEA em Santa Catarina, identificando uma prevalência de 1,31 de autistas a cada 10.000 pessoas $^{4}$ e outro em que estimaram uma prevalência de $0,88 \%$ de casos de TEA em uma população de 1.470 crianças do município de Atibaia em São Paulo ${ }^{5}$.

Ao mesmo tempo, nos últimos anos, importantes conquistas ocorreram em prol das pessoas com TEA no âmbito das políticas públicas no Brasil, como a criação da Rede de Atenção Psicossocial (RAPS), que compartilha dos princípios e diretrizes do Sistema Único de Saúde (SUS), através da Portaria no 3088, de 23 de dezembro de 2011. A RAPS estabelece pontos de atenção para atendimento de pessoas com problemas mentais, sendo composta equipamentos e serviços variados, como os Centros de Atenção Psicossocial (CAPS) em suas diferentes modalidades. Segundo as diretrizes do Ministério da Saúde ${ }^{6,7}$ os CAPS e CAPSi são equipamentos designados para o tratamento do autismo ${ }^{8}$. Além disso, a instituição da Política Nacional de Proteção dos Direitos da Pessoa com Transtorno do Espectro Autista, através da lei 12.764, de 11 de dezembro de 2012, estabelece que o indivíduo com TEA é considerado pessoa com deficiência, para todos os efeitos legais.

Apesar desses avanços, a dificuldade de acesso a serviços de saúde para atendimento a pessoas com Autismo tem sido apontada como um dos fatores contribuintes para a diminuição da qualidade de vida, o aumento do estresse dos cuidadores, a postergação da definição diagnóstica e do estabelecimento do tratamento adequado, configurando um desafio para Saúde Mental ${ }^{9,10}$. Estas dificuldades somadas a barreiras de ordem cultural, econômica, geográfica e funcional estão espelhadas na busca por informações que resultem, de fato, em solução ${ }^{11}$. Dado que a população do município do Rio de Janeiro são pouco mais de 6 milhões e 1 a $2 \%$ destes apresentariam o TEA $^{12}$, pode-se estimar que a demanda por informação e acesso ao tratamento seja significativa, não sendo mais possível negligenciá-la.

É dado que a informação é, segundo a teoria da análise de redes sociais (ARS), recurso inestimável na busca por soluções. Há de se considerar que as pessoas busquem em suas redes pessoais, impulsos para o alcance de tais recursos, como o acesso a serviços de saúde ${ }^{13}$.

A teoria da ARS no Brasil tem sido utilizada nos últimos anos em investigações no campo da saúde coletiva, permitindo a construção de instrumentos indutivos para a investigação social, que visam identificar as relações existentes entre atores de uma rede e mensurar sistematicamente o comportamento social dos mesmos ${ }^{14}$. Isso permite compreender as interdependências e assimetrias existentes em toda ação coletiva ${ }^{15}$.

Atualmente, o interesse pelas redes sociais ganha evidência na avaliação de serviços de saúde, como instrumento aos programas de saúde ${ }^{16}$, entre outros, inclusive na área da Saúde Mental ${ }^{17,18}$.

A internet, como espaço de interações múltiplas favorecendo a ligação entre as pessoas e seus interesses tem, portanto, se constituído importante fonte de acesso à informação sobre a saúde. As coletividades vêm se organizando ao redor de classificações biomédicas e se apropriando, cada vez mais, deste espaço virtual ${ }^{19}$, que espelha a demanda por soluções. Urge, portanto, verificar como tais caminhos têm se relacionado aos desfechos, contribuindo com a melhoria do acesso a serviços de saúde. Dessa forma, o presente artigo tem como objetivo analisar a rede virtual de acesso a informações sobre atendimento para Autismo no município do Rio de Janeiro, através da perspectiva da Análise de Rede Sociais.

\section{Procedimentos metodológicos}

Busca-se analisar a rede de informações virtuais existentes sobre os locais de atendimento para pessoas com TEA no município do Rio de Janeiro, no ano de 2017, a partir da análise das ligações entre fontes virtuais e locais de atendimento citados por essas fontes. A coleta de dados realizouse pela procura na ferramenta de busca Google, sem a utilização de ferramentas adicionais e sem o uso de aspas, com apenas a seguinte combinação de palavras, visto que o intuito do estudo não é aprofundar possibilidades de termos, mas de fazer uma primeira exploração, ou exame, da atual situação: "Atendimento para Autismo no município do Rio de Janeiro". Esta forma de busca foi planejada com o intuito de deixá-la o mais 
aberta possível, sem exigência de habilidades específicas, sendo intuitiva, intentando resultados mais aproximados da pesquisa de um cidadão em busca de informação para um caso que o aflige, como se o pesquisador fizesse parte deste grupo de internautas leigos ${ }^{19}$. Outra medida para evitar a influência da personalização de resultados foi a realização da busca em um equipamento (PC, ou IP) não utilizado pelos pesquisadores, conforme aponta Pariser ${ }^{20}$ sobre a influência do "filtro invisível" nas buscas em internet. Estas medidas tiveram o intuito de maximizar a confiabilidade nos resultados almejados.

Foram coletados os seguintes dados através de uma ficha construída para tal finalidade e posterior armazenamento e organização em Excel: (a) nome da fonte de informação (sites, páginas do Facebook, blogs, etc.) e (b) nome dos locais de atendimento mencionados em cada fonte e o tipo de serviço (Serviços públicos, privados com fins lucrativos e ONGs). Os locais de atendimento foram divididos em 3 grupos de acordo com seu tipo para melhor o entendimento da análise dos dados (Quadro 1). A coleta de dados foi en-

Quadro 1. Grupos de locais de atendimento

\begin{tabular}{|l|l|}
\hline Grupos & Tipo de serviço \\
\hline Grupo 1 & $\begin{array}{l}\text { Serviços públicos (CAPSi, institutos, } \\
\text { ambulatórios especializados e Centros } \\
\text { Especializados em Reabilitação } \\
\text { Intelectual e Autismo) }\end{array}$ \\
\hline Grupo 2 & $\begin{array}{l}\text { Serviços particulares que fazem parte } \\
\text { do setor privado com fins lucrativos } \\
\text { (Clínicas e consultórios particulares). }\end{array}$ \\
\hline Grupo 3 & Organizações não governamentais. \\
\hline
\end{tabular}

cerrada no momento em que as informações se desviaram do foco da pesquisa, como por exemplo, serviços de saúde fora no município do Rio de Janeiro ou serviços que não fossem para a assistência de pessoas com TEA.

A coleta dos dados foi realizada em 17/09/2017, conforme se tem empregado na busca por informações virtuais ${ }^{21}$. Posteriormente, foram inseridos, através de uma tabela de arestas, no software Gephi versão 0.9.1, de livre acesso, para a construção dos sociogramas, permitindo visualização e exploração destes gráficos. Este software é amplamente utilizado em pesquisas de $\mathrm{ARS}^{22-24}$. Aos moldes dos pacotes estatísticos, ele elabora gráficos a partir das variáveis (os indivíduos, seus atributos, locais e/ou instituições), construindo um banco de dados no Excel exportado ao referido software. Subsequentemente, obtém-se os sociogramas para a análise ${ }^{25,26}$. Foram calculadas métricas, posteriormente utilizadas para a análise dos dados. As métricas de interesse selecionadas foram: Centralidade de Grau, Centralidade de Intermediação e Densidade. O Quadro 2 as apresenta, acompanhadas de seus significados ${ }^{27,28}$.

\section{Resultados}

O total de resultados obtidos na busca foi de 4.020, sendo verificadas as páginas até a lista de opções ofertadas não ter mais pertinência, o que só ocorreu na $4^{a}$ página.

Verificou-se que o sociograma resultante do uso do software Gephi se caracterizou por uma predominância de fontes virtuais que fazem parte do grupo 3 e a maioria dos locais citados faz parte do grupo 1 e 3, conforme detalhado na tabela 1 e no sociograma 1 . A Tabela 1 apresen-

Quadro 2. Métricas da ARS utilizadas pelo estudo.

\begin{tabular}{|l|l|}
\hline \multicolumn{1}{|c|}{ Métricas } & \multicolumn{1}{c|}{ Significado } \\
\hline Centralidade de Grau & $\begin{array}{l}\text { A Centralidade caracteriza-se pelo “nó" ou ator, do qual parte a maioria das } \\
\text { relações. A centralidade de grau agrega maior controle e poder. }\end{array}$ \\
\hline Centralidade de Intermediação & $\begin{array}{l}\text { A medição de quanto um nó pode controlar/mediar o fluxo de informações, } \\
\text { dada sua posição na rede; nós com centralidade de intermediação altas } \\
\text { podem ser considerados como articuladores do fluxo da rede. }\end{array}$ \\
\hline Densidade & $\begin{array}{l}\text { A densidade é a porcentagem de laços que existe em uma rede dentre o } \\
\text { total de laços possíveis. Uma densidade igual a 1.0 em uma rede equivale } \\
\text { dizer que se trata de uma rede densa onde todos se relacionam com todos, } \\
\text { utilizando } 100 \% \text { do seu potencial; uma densidade zero equivale à ausência } \\
\text { de ligações, ninguém se conhece, não há relações estabelecidas, inexistência } \\
\text { de rede. }\end{array}$ \\
\hline
\end{tabular}

Fonte: Com base em artigos a respeito ${ }^{25-27}$ 
Tabela 1. Descrição e métricas utilizadas para a elaboração do sociograma da Figura 1.

\begin{tabular}{lr}
\hline \multicolumn{2}{c}{ Características Gerais } \\
\hline Número de nós & 47 \\
Número de laços & 66 \\
\hline \multicolumn{2}{c}{ Fontes Virtuais } \\
\hline Número de fontes virtuais originadas de \\
ONGs \\
Número de fontes virtuais originadas de \\
Serviços Públicos \\
Número de fontes virtuais originadas de \\
Serviços particulares \\
Número de fontes virtuais originadas de \\
Imprensa \\
Total \\
\hline \multicolumn{2}{c}{ Instituições Citadas } \\
\hline ONGs & 14 \\
Serviços públicos & 12 \\
Serviços particulares com fins lucrativos & 9 \\
Total & 33 \\
\hline \multicolumn{2}{c}{ Métricas } \\
\hline Grau médio & 2,809 \\
Densidade & 0,061 \\
\hline
\end{tabular}

ta a descrição geral e métricas utilizadas para a elaboração do sociograma (densidade, o grau de intermediação e o grau médio).

Conforme o observado, o número total de fontes virtuais estudadas foi 14. O total de fontes originadas de ONGs foi de 7, sendo 3 relativas aos serviços particulares com fins lucrativos, 2 procedentes de órgãos públicos e 2 originados da impressa. O número total de Instituições/locais de atendimento citados nas fontes virtuais foi de 33. Desses, 12 eram ONGs (grupo 3), outros 12 eram serviços públicos (grupo 1) e 09 serviços particulares (grupo 2).

Sobre as métricas calculadas mostradas na Tabela 1, a densidade geral da rede foi de 0,061, ou seja, a rede estabelece cerca de $6,1 \%$ de todas as relações possíveis de serem realizadas, configurando uma rede com baixa densidade. $\mathrm{O}$ grau médio da rede foi de 2,809, sendo que o nó com maior grau de centralidade foi uma fonte virtual originada de uma ONG, como pode ser visto no sociograma da Figura 1.

O sociograma da Figura 1 representa a rede de informações dispostas virtualmente sobre busca por atendimento para TEA no município do Rio de Janeiro, no momento desta pesquisa, através de ligações entre fontes virtuais e insti- tuições/ locais de atendimento, de acordo com o tipo (serviço privado, público ou ONGs). Conforme o observado, o ator que demonstrou maior centralidade na rede é uma fonte virtual originada de uma ONG (grupo 3) e em segundo lugar uma fonte virtual originada de um serviço privado (grupo 2). Além disso, a maioria dos locais de atendimento citados pela fonte virtual com maior centralidade faz parte do grupo 2 e 3 .

Quanto à interação intergrupos, observou-se um grau de isolamento do grupo 1 na rede, evidenciado por um baixo número de conexões do grupo 1 (locais de atendimento da rede pública) com os demais grupos. Os grupos 2 e 3 apresentam maior número de conexões entre eles, ou seja, compartilham locais citados entre eles.

\section{Discussão}

Mesmo considerando que a utilização dos recursos da internet na busca por informações leve a resultados distintos em função de uma plêiade de elementos dinâmicos e mutáveis, ela expressa relações, contatos e movimentos reais. Certamente, consideramos aqui, à moda de estudos clássicos transversais da epidemiologia, que esta rede se apresenta como uma "janela no tempo", contudo, espelhando verdadeiros caminhos realizados. Nesta lógica reconhece-se a limitação do estudo se analisam os resultados, não obstante a possibilidade de proposições mais generalistas, já que são conteúdos compartilhados pelo usuário e armazenados pelo provedor ${ }^{19}$, logo passíveis, ao longo do tempo, de reutilização, dispersão e abrangência.

Os resultados encontrados sugerem que as fontes virtuais originadas de ONGs exercem importante influência no fluxo de informação disponível virtualmente sobre atendimento para Autismo, pois compõe a grande parte da rede e têm maior grau de centralidade (Figura 1). Quando um ator tem centralidade, possui maior número de ligações que os demais integrantes, o que lhe confere maior acesso a informações e recursos de diversas ordens ${ }^{29}$. A centralidade viabiliza controle e poder, influenciando nas tomadas das decisões ${ }^{30}$, e possivelmente servirão como guias aos que buscam informações sobre o TEA. Importa, portanto, que sejam bons "faróis”. Já que as ONGs parecem exercer importante papel, intermediando informações a respeito de tratamento e serviços, lhes conferindo papel de mediação central ${ }^{25}$, seria pertinente monitorá -las, conjugando-as oficialmente em uma rede de 


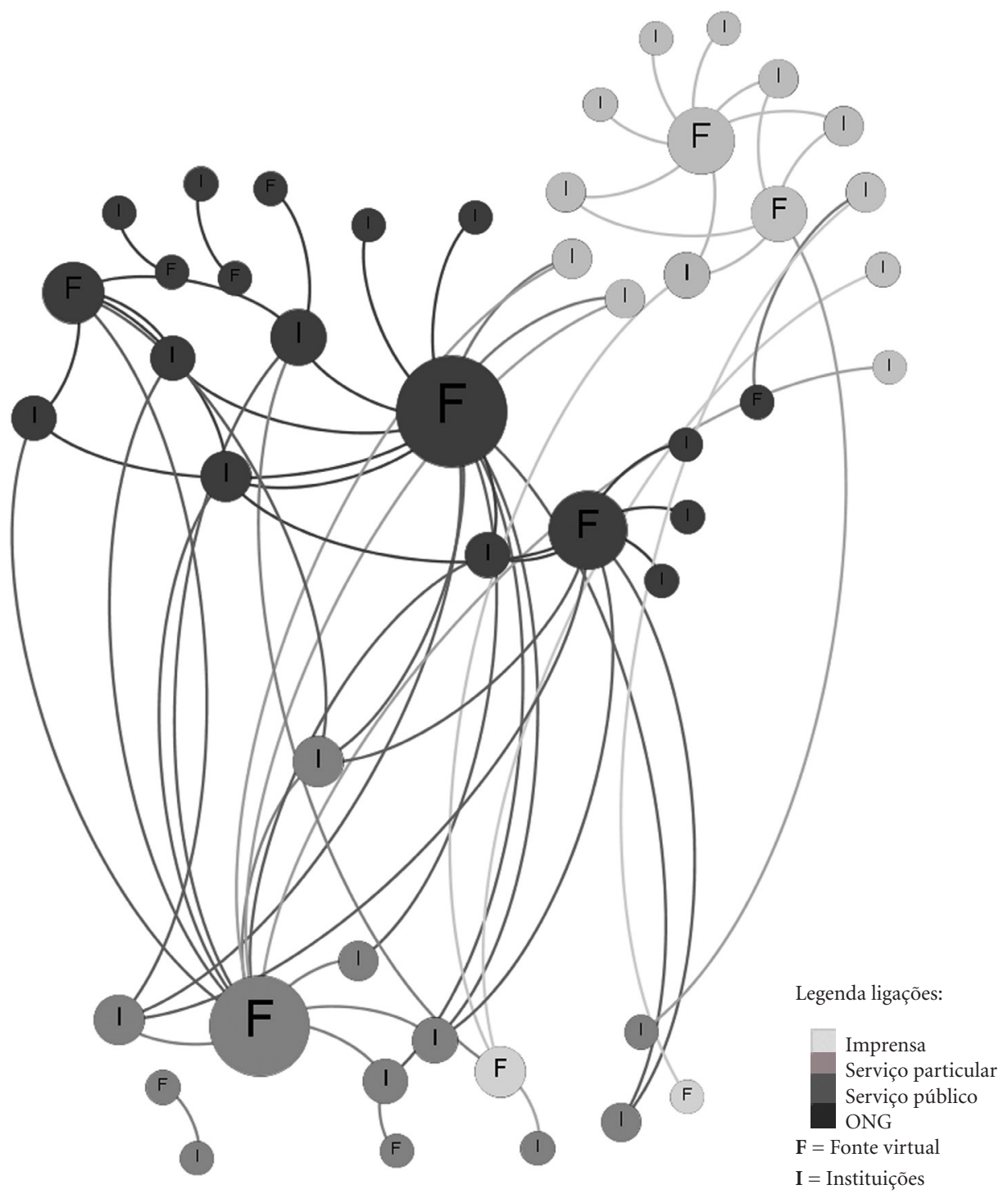

Figura 1. Sociograma representativo da rede de informações virtuais sobre atendimento para Autismo no município do Rio de Janeiro.

parcerias institucionalizadas, de modo a agregar e não de abster-se delegando.

As Instituições filantrópicas ou não governamentais vêm tendo protagonismo na construção de políticas públicas brasileiras para o Autismo, e a atual divergência existente entre duas frentes: atenção psicossocial e o campo da reabilitação, representados pelas associações de pais e familiares de pessoas com Autismo ${ }^{31,32}$. Desta maneira, é possível perceber que as características observadas nesta rede podem demonstrar o afastamento do que é preconizado pelo SUS. Este caracterizase por um modelo de atenção a partir de serviços de base comunitária, seguindo os princípios da intersetorialidade, de rede, território, acolhimento universal e encaminhamento. Tal organização destinar-se-ia a possibilitar a atenção integral de pessoas com autismo e outros transtornos men- 
tais, que têm como principal local de atendimento a esta população o CAPS e o CAPSi ${ }^{7}$, os quais não parecem ter a centralidade planejada.

A rede possui uma baixa densidade. A densidade é a porcentagem de laços que existe em uma rede dentre o total de laços possíveis ${ }^{26}$. Uma rede com maior densidade tende a apresentar melhor fluxo de capital social, que gerado nas relações pode ser utilizado pela estrutura social em prol de seu próprio benefício ${ }^{29}$. No entanto, a baixa densidade encontrada na rede de informações disponíveis virtualmente sobre atendimento para TEA é uma característica esperada, já que o propósito dela é ser auxilio de caminho e de destinação aos serviços de saúde. Neste caso, não resulta negativamente. Entretanto, se estivesse incorporada à uma estratégia governamental, talvez pudesse gerar mais contatos e, consequentemente, maior dispersão e difusão de informação na rede, beneficiando um maior público.

$\mathrm{Na}$ rede da Figura 1, os grupos 2 e 3 têm maior número de locais citados entre eles, demonstrando maior interação entre esses grupos. Os locais de atendimento do grupo 1 são pouco citados nos outros grupos de fontes virtuais, além de não indicarem locais de atendimento dos outros grupos, permitindo considerar certo grau de isolamento dos serviços públicos, ao menos por estes caminhos virtuais analisados. Ou seja, isso sugere que as fontes virtuais em maior número na rede e que têm maior centralidade, fornecem listas de locais de atendimento, que em sua maioria não incluem os locais de saúde da rede pública. Se estas possuem maior centralidade devido ao acesso as listas, pode-se ponderar que há uma busca por informação relacionada ao acesso aos serviços. Além disso, as fontes virtuais de origem governamental não mencionam locais de atendimento que não façam parte da linha de cuidado preconizada pelo SUS, o que, apesar de lógico, espelha as políticas públicas a este respeito. Por outro lado, representam caminhos espontâneos relacionais construídos pelos indivíduos na busca por soluções. Estes movimentos acabam por gerar novas estruturas, que, por sua constância e resolutividade, tornam-se normativas ${ }^{25}$.

Uma possível explicação para o panorama observado pode estar relacionada a um dos desafios no setor específico da saúde mental infantil e juvenil, que é, ainda, a necessidade de expansão dos diferentes serviços que compõem a rede de cuidados e o aumento do número de CAPSi, ambulatórios e outros dispositivos de saúde mental, principalmente, aqueles voltados para infância e adolescência ${ }^{33}$. Somada a essa situação, o aumen- to considerável na prevalência de TEA na população nos últimos anos ${ }^{34}$ gera uma maior demanda de atendimento, configurando um desafio para a Saúde Mental infanto-juvenil na cidade do Rio de Janeiro.

Sabe-se que o diagnóstico e o tratamento precoce para TEA pode prever o prognóstico e a evolução do tratamento ${ }^{35}$. Além disso, a rotina dos cuidadores tendo que lidar com os sintomas comportamentais pode ser extremamente estressante para a família. Desta forma, como evidenciado acima, essas famílias constroem seus próprios caminhos e se organizam virtualmente, na busca de informações e soluções ${ }^{18}$, diante da dificuldade de acesso às informações e aos serviços de saúde.

Desta forma, o panorama observado na rede virtual de informações sobre atendimento para Autismo no município do Rio de Janeiro, demonstra não ser favorável para o acesso à informações sobre o atendimento na rede pública de saúde, podendo influenciar no acesso aos serviços dessa rede e nas escolhas de tratamento. Essa rede de informações virtuais pode sugerir a adaptação da sociedade a ausência de ações que cabem ao estado, proporcionando atendimento integral e serviços acessíveis universalmente. A dificuldade de acesso aos serviços de saúde vem sendo apontada em estudos internacionais. Estudos amerianos apontam que crianças com TEA têm uma maior probabilidade de apresentar uma necessidade de terapia não atendida, em comparação com crianças com outras transtornos no desenvolvimento com o $\mathrm{TDAH}^{36}$. As crianças com TEA requerem serviços de apoio, como terapia ocupacional, fisioterapia e fonoaudiológia, mas são 1,4 vezes mais propensas a relatar uma necessidade não atendida de terapia ,em comparação com outras outras necessidades especiais ${ }^{37}$.

Percebe-se a importância da melhor visibilidade, ocupação e apropriação dos espaços virtuais por parte das entidades governamentais, tendo em vista sua forte influência como meio de acesso a informação. Seria aconselhável a compreensão dos fluxos de informação e o papel dos diversos atores envolvidos nas redes de modo mais amplo, para a elaboração de políticas públicas de acesso, inclusão e geração de bem-estar, incorporando ferramentas que se mostram úteis e sem custos adicionais. $\mathrm{O}$ acesso à informação, principalmente aquele existente fora do grupo, é o elementochave para a investigação. As ações políticas não podem ignorar informações que os próprios grupos elaboram a partir de seus espaços de vivências e interação ${ }^{38}$. Sendo assim, seria recomen- 
dável a incorporação das Associações/Organizações comunitárias na rede de atendimentos, a fim de favorecer a comunicação entre os diferentes serviços e o acesso aos serviços de atendimento, mostra-se uma estratégia acessível e prática, cujos caminhos virtuais se mostram viáveis.

\section{Conclusão}

Através da perspectiva da Análise de Redes Sociais foi possível concluir que os caminhos virtuais de acesso a informações sobre atendimento para TEA no município do Rio de Janeiro apontam um importante protagonismo e organização da comunidade na busca pela solução deste problema, refletindo a dificuldade de acesso às informações e, principalmente, aos serviços da rede pública de saúde, incutindo a perspectiva de que este caminho virtual seja incorporado às ações governamentais em prol de oportuna, viável e acessível solução àqueles que buscam por serviços dedicados ao TEA.

\section{Colaboradores}

LP Rossi participou da elaboração do estudo, coleta de dados, análise dos dados e elaboração do texto. M Gomide e L Abelha participaram da elaboração do estudo, análise de dados e elaboração do texto. GM Lovisi participou na parte da análise dos dados e elaboração do texto.

\section{Referências}

1. Christensen DL, Baio J, Braun KV, Bilder D, Charles J, Constantino JN, Daniels J, Durkin MS, Fitzgerald RT, Kurzius-Spencer M, Lee LC, Pettygrove S, Robinson C, Schulz E, Wells C, Wingate MS, Zahorodny W, Yeargin-Allsopp M. Prevalence and characteristics of autism spectrum disorder among children aged 8 years: autism and developmental disabilities monitoring network, 11 sites, United States, 2012. Surveill Summ 2016; 65(3):1-23.

2. Xu G, Strathearn L, Liu B, Bao W. Prevalence of autism spectrum disorder among US children and adolescents, 2014-2016, United States. JAMA 2018; 319(1):81-82.

3. Teixeira MCTV, Mecca TP, Velloso RL, Bravo RB, Ribeiro SHB, Mercadante MT, Paula CS. Literatura científica brasileira sobre transtornos do espectro autista. Rev Ass Med Bras 2010; 56(5):607-614.

4. Ferreira E. Prevalência de autismo em Santa Catarina: uma visão epidemiológica contribuindo para a inclusão social [dissertação]. Florianópolis: Universidade Federal de Santa Catarina; 2008.

5. Ribeiro SHB. Prevalência dos transtornos invasivos do desenvolvimento no município de Atibaia: um estudo piloto [dissertação]. São Paulo: Universidade Presbiteriana Mackenzie; 2007.

6. Brasil. Ministério da Saúde (MS). Reforma psiquiátrica e política de saúde mental no Brasil. In: Relatório da Conferência Regional de Reforma dos Serviços de Saúde Mental: 15 Anos Depois de Caracas. Brasília: MS; 2005. p. 1-56.

7. Brasil. Ministério da Saúde (MS). Conheça a RAPS: rede de Atenção Psicossocial. Brasília: MS; 2013.
8. Nunes F, Ortega F. Ativismo político de pais de autistas no Rio de Janeiro: reflexões sobre o "direito ao tratamento". Saude Soc 2016; 25(4):964-975.

9. Gomes PTM, Lima LHL, Bueno MKG, Araújo LA, Souza NM. Autismo no Brasil: desafios familiares e estratégias de superação: revisão sistemática. J Pediatr 2015; 91(2):111-121.

10. Favero-Nunes MA, Santos MA. Itinerário terapêutico percorrido por mães de crianças com transtorno autístico. Psicol Reflex Crit 2010; 23(2):208-221.

11. Falavina OP, Cerqueira MB. Saúde mental infanto-juvenil: usuários e suas trajetórias de acesso aos serviços de saúde. Espaç Saude 2008; 10(1):34-46.

12. Centers for Disease Control and Prevention [Internet]. Atlanta: CDC; 2016. Data and statistics; [about 3 screens]. [acessado 2018 jan 05]. Disponível em: https://www.cdc.gov/ncbddd/autism/data.html

13. Portugal S. Quanto vale o capital social: o papel das redes informais na provisão de recursos. In: Fontes B, Martins $\mathrm{PH}$, organizadores. Redes, práticas associativas e gestão pública. Recife: Ed. UFPE; 2006. p. 51-74.

14. Gomide M, Grossetti M. Rede social e desempenho de programas de saúde: uma proposta investigativa. Physis 2010; 20(3):873-893.

15. Varanda M. Acção colectiva entre pequenos empresários: uma análise de redes sociais. Anal Social 2007; 42(182):207-230.

16. Valente TW, Palinkas LA, Czaja S, Chu KH, Brown CH. Social network analysis for program implementation. PLoS One 2015; 10(6):e0131712. 
17. Mesquita RB, Landim FLP, Collares PM, Luna CG. Análise de redes sociais informais: aplicação na realidade da escola inclusiva. Interface (Botucatu) 2008; 12(26):549-562.

18. Portugal S, Nogueira C, Hespanha P. As teias que a doença tece: a análise das redes sociais no cuidado da doença mental. Dados 2014; 57(4):935-968.

19. Ortega F, Zorzanelli R, Meierhoffer LK, Rosário CA, Almeida CF, Andrada BFCC, Chagas BS, Feldman C. A construção do diagnóstico do autismo em uma rede social virtual brasileira. Interface (Botucatu) 2013; 17(44):119-132.

20. Pariser, E. O filtro invisível: o que a internet está escondendo de você. Rio de Janeiro: Zahar; 2012.

21. Coutinho T, Esher AF, Osorio-de-Castro CGS. Mapeando espaços virtuais de informação sobre TDA/H e usos do metilfenidato. Physis 2017; 27(3):749-769.

22. Costa PHA, Martins LF, Medeiros AX, Salgado JA, Silva D, Maurício W, Ronzani T M, Colugnati FAB. Sistema de referência e de contrarreferência na rede de atenção aos usuários de drogas: contribuições da análise de redes sociais. Cad Saude Col 2015; 23(3):245-252.

23. Claudia PM, Marta LT. Metodología basada en el enfoque de redes y uso del software Gephi: el caso de AIDER en el fortalecimiento del capital social para el Manejo Forestal Comunitario en la cuenca de Aguaytía, Ucayali, Perú. RISTI 2016; (19):131-144.

24. Kuz A, Falco M, Giandini R. Análisis de redes sociales: un caso práctico. Comp y Sist 2016; 20(1):89-106.

25. Borgatti SP. Centrality and network flow. Soc Network 2005; 27(1):55-71.

26. Vaz GJ. A construção dos sociogramas e a teoria dos grafos. Rev Bras Psicodrama 2009; 17(2):67-78.

27. Mccarty C. La estrutura em las redes personales. Redes 2010; 19(11):242-271.

28. Gomide M, Schütz GE, Carvalho MAR, Câmara VM. Fortalezas, oportunidades, fraquezas e ameaças (matriz FOFA) de uma comunidade ribeirinha sul-amazônica na perspectiva da análise de redes sociais: aportes para a atenção básica à saúde. Cad Saude Col 2015; 23(3):222-230.

29. Granovetter MS. The strength of weak ties. Am J Sociol 1973; 78(6):1360-1380.

30. Lino CRG, Gomide M. Organização do serviço e análise de redes sociais: estudo de caso na vigilância em saúde ambiental. Physis 2015; 25(2):443-465.
31. Oliveira BDC, Feldman C, Couto MCV, Lima RC. Políticas para o autismo no Brasil: entre a atenção psicossocial e a reabilitação. Physis 2017; 27(3):707-726.

32. Ortega F. Deficiência, autismo e neurodiversidade. Cienc Saude Colet 2009; 14(1):67-77.

33. Couto MCV, Duarte CS, Delgado PGG. A saúde mental infantil na saúde pública brasileira: situação atual e desafios. Rev Bras Psiquiatr 2008; 30(4):384-389.

34. Christensen DL, Bilder DA, Zahorodny W, Pettygrove S, Durkin MS, Fitzgerald RT, Rice C, Kruzius-spencer M, Baio J, Yeargin-allsopp M. Prevalence and characteristics of autism spectrum disorder among 4-yearold children in the autism and developmental disabilities monitoring network. J Dev Behav Pediatr 2016, 37(1):1-8.

35. Oliveira G. Autismo: diagnóstico e orientação parte i: vigilância, rastreio e orientação nos cuidados primários de saúde. Acta Pediatr Port 2009; 40(6):278-287.

36. Benevides TW, Carretta HJ, Ivey CK, Lane SJ. Therapy access among children with autism spectrum disorder, cerebral palsy, and attention-deficit-hyperactivity disorder: a population-based study, Dev Med Child Neurol 2017; 59(12):1291-1298

37. Benevides TW, Carretta HJ, Lane SJ. Unmet need for therapy among children with autism spectrum disorder: results from the 2005-2006 and 2009-2010 National Survey of Children with Special Health Care Needs. Matern Child Health J 2016; 20(4):878-888.

38. Marteleto RMS, Silva ABO. Redes e capital social: o enfoque da informação para o desenvolvimento local. $C i$ Inf 2004; 33(3):41-49.

Artigo apresentado em 31/01/2018

Aprovado em 06/03/2018

Versão final apresentada em 24/05/2018 